\title{
Global Bifurcations in a Three-Dimensional Financial Model of Bull and Bear Interactions
}

\author{
Fabio Tramontana, Laura Gardini, Roberto Dieci, and Frank Westerhoff
}

\section{Introduction}

In a previous paper Tramontana et al. (2009), we developed a three-dimensional discrete-time dynamic model in which two stock markets of two countries, say $\mathrm{H}$ (ome) and $\mathrm{A}$ (broad), are linked via and with the foreign exchange market. The latter is modelled in the sense of Day and Huang (1990), i.e. it is characterized by a nonlinear interplay between technical traders (or chartists) and fundamental traders (or fundamentalists). In the absence of connections, the foreign exchange market is driven by the iteration of a one-dimensional cubic map, which has the potential to produce a regime of alternating and unpredictable bubbles and crashes for sufficiently large values of a key parameter, which captures the speculative behavior of chartists. Such a dynamic feature, first observed and explained by Day and Huang (1990) in their stylized model of financial market dynamics, can be understood with the help of bifurcation analysis: an initial situation of bi-stability (two coexisting, attracting non-fundamental steady states around an unstable fundamental equilibrium) evolves into coexistence of cycles or chaotic intervals within two disjoint bull and bear regions, which eventually merge via a homoclinic bifurcation. By introducing connections between markets (i.e. by allowing stock market traders to be active abroad), the endogenous fluctuations originating in one of the markets spread throughout the whole three-dimensional system. It therefore becomes interesting to investigate how the coupling of the markets affects the bull and bear dynamics of the model. With regard to this, in Tramontana et al. (2009) we already performed a thorough analytical and numerical study of two simplified lower-dimensional cases, where connections are either totally absent (each market evolves according to an independent one-dimensional map) or occur in one direction (a two-dimensional system evolves independently of the third dynamic equation). Also a short analysis of the stability of the equilibria of the three-dimensional model was there started, arguing that the global (homoclinic) bifurcations may still be a characteristic of the

F. Tramontana $(\bowtie)$

Università di Ancona, Dipartimento di Economia Piazza Martelli, 860121 Ancona, Italy, e-mail: f.tramontana@univpm.it 
dynamics. This investigation is precisely the object of the present paper. We shall analyze the dynamic behavior of the complete three-dimensional model, following the approach adopted in Tramontana et al. (2009), based mainly on the numerical and graphical detection of the relevant global bifurcations. Although analytical conditions for such global bifurcation, mainly homoclinic bifurcations, are difficult to be formalized, their existence and occurrence can be numerically detected. As it is standard in the qualitative study of dynamic behaviors, the transverse crossing between stable and unstable sets of unstable cycles, leading to homoclinic trajectories, give numerical tools which may be considered as proofs in a given numerical example.

The structure of the paper is as follows. In Sect. 2 we briefly describe the threedimensional model of interacting stock and foreign exchange markets. The main results regarding the lower-dimensional subcases explored in Tramontana et al. (2009) are summarized in Sect. 3. Section 4 deals with the dynamics of the complete three-dimensional model by discussing, in particular, the steady state properties and the existence of multiple equilibria (Sect. 4.1), the homoclinic bifurcations of the non-fundamental steady states (Sect.4.2) and of the fundamental equilibrium (Sect. 4.3), and the so-called final bifurcation (Sect.4.4). Section 5 concludes the paper.

\section{The Dynamic Model}

This model describes the joint evolution of two stock markets (denoted as $H$ and $A$ ), denominated in different currencies, and the related foreign exchange market. While the two stock prices $\left(P_{t}^{H}\right.$ and $P_{t}^{A}$, respectively) adjust over time depending on the excess demand for stock generated by national and foreign fundamental traders, the exchange rate ${ }^{1}\left(S_{t}\right)$ depends on the excess demand of currency $H$. The latter consists of $(i)$ demand for currency by heterogeneous speculators (technical and fundamental traders) who explicitly focus on the foreign exchange market and (ii) demand for currency by stock market traders who invest abroad, who obviously buy/sell foreign currency to conduct stock market transactions. In the following, we denote as $F^{H}, F^{A}$ and $F^{S}$ the fundamental values of the two stock prices and the exchange rate, respectively. Assuming, for the sake of simplicity, a linear price impact function, prices in the three markets jointly evolve according to the following laws of motion:

$$
\begin{gathered}
P_{t+1}^{H}=P_{t}^{H}+a^{H}\left(D_{F, t}^{H H}+D_{F, t}^{H A}\right), \\
P_{t+1}^{A}=P_{t}^{A}+a^{A}\left(D_{F, t}^{A A}+D_{F, t}^{A H}\right),
\end{gathered}
$$

\footnotetext{
${ }^{1}$ Here we define the exchange rate as the price, expressed in currency $A$, of one unit of currency $H$.
} 


$$
S_{t+1}=S_{t}+d\left(P_{t}^{H} D_{F, t}^{H A}-\frac{P_{t}^{A}}{S_{t}} D_{F, t}^{A H}+D_{C, t}^{S}+D_{F, t}^{S}\right),
$$

where $a^{H}, a^{A}$ and $d$ are positive parameters, and where the demand terms appearing on the right-hand sides of the above equations have the following definitions and meaning:

- $D_{F, t}^{H H}=b^{H}\left(F^{H}-P_{t}^{H}\right), b^{H}>0$, is the demand ${ }^{2}$ for stock $H$ by the fundamental traders (or fundamentalists) from country $H$.

- $D_{F, t}^{H A}=c^{H}\left[\left(F^{H}-P_{t}^{H}\right)+\gamma^{H}\left(F^{S}-S_{t}\right)\right], c^{H}=0, \gamma^{H}>0$, is the demand for stock $H$ by the fundamental traders from country $A$.

- $D_{F, t}^{A A}=b^{A}\left(F^{A}-P_{t}^{A}\right), b^{A}>0$, is the demand for stock $A$ by the fundamental traders from country $A$.

- $D_{F, t}^{A H}=c^{A}\left[\left(F^{A}-P_{t}^{A}\right)+\gamma^{A}\left(\frac{1}{F^{S}}-\frac{1}{S_{t}}\right)\right], c^{A}=0, \gamma^{A}>0$, is the demand for stock $A$ by the fundamental traders from country $H$.

- $D_{C, t}^{S}=e\left(S_{t}-F^{S}\right), e>0$, and $D_{F, t}^{S}=f\left(F^{S}-S_{t}\right)^{3}, f>0$, are the demands of currency $H$ by chartists and fundamentalists, respectively, who enter speculative positions in the foreign exchange market. In particular, chartist demand coefficient, $e$, turns out to be an important bifurcation parameter in our analysis.

The following additional comments about agents' demands are useful:

(1) Fundamentalists seek to profit from mean reversion, so that they submit buying orders (positive demand) when the market is undervalued (the price is below fundamental) and selling orders (negative demand) when the market is overvalued.

(2) In addition, foreign fundamentalists may also benefit from exchange rate movements, and therefore their demand function also includes a term that is dependent on the observed mispricing in the foreign exchange market; in particular, traders from $H$ to $A$ take into account the reciprocal values of the exchange rate and its fundamental.

(3) In the foreign exchange market, chartists believe in the persistence of bull markets or bear markets and therefore optimistically buy (pessimistically sell) currency $H$ as long as the exchange rate is high (low). Fundamentalists seek to exploit misalignments using a nonlinear trading rule. As long as the exchange rate is close to its fundamental value, fundamentalists are relatively cautious, but the greater the mispricing, the more aggressive they become.

(4) Finally, $P_{t}^{H} D_{F, t}^{H A}$ represents the demand for currency $H$ generated by stock market orders from $A$ to $H$, and symmetrically $P_{t}^{A} D_{F, t}^{A H}$ is the demand for currency $A$ generated by stock market orders from $H$ to $A$ : the latter is

\footnotetext{
${ }^{2}$ The demand for stock is given in real units.
} 
converted into an amount of currency $\mathrm{H}$ of opposite sign, via the reciprocal exchange rate $\frac{1}{S_{t}}$.

By specifying all of the demand terms in (1)-(3), we obtain a three-dimensional dynamical system with the following structure

$$
\left\{\begin{array}{l}
P_{t+1}^{H}=G^{H}\left(P_{t}^{H}, S_{t}\right), \\
S_{t+1}=G^{S}\left(P_{t}^{H}, S_{t}, P_{t}^{A}\right), \\
P_{t+1}^{A}=G^{A}\left(S_{t}, P_{t}^{A}\right) .
\end{array}\right.
$$

In particular, for $c^{H}=c^{A}=0$ the structure of the system (4) simplifies into three independent dynamic equations, $P_{t+1}^{H}=G^{H}\left(P_{t}^{H}\right), S_{t+1}=G^{S}\left(S_{t}\right), P_{t+1}^{A}=$ $G^{A}\left(P_{t}^{A}\right)$, of which that for exchange rate $S$ is nonlinear (of cubic type), whereas the two stock prices $P^{H}$ and $P^{A}$ evolve linearly. More interestingly, for $c^{A}=0$ but $c^{H}>0$ the system is of the type

$$
\left\{\begin{array}{l}
P_{t+1}^{H}=G^{H}\left(P_{t}^{H}, S_{t}\right), \\
S_{t+1}=G^{S}\left(P_{t}^{H}, S_{t}\right), \\
P_{t+1}^{A}=G^{A}\left(P_{t}^{A}\right),
\end{array}\right.
$$

that is to say, $P^{A}$ decouples from the system, whereas $P^{H}$ and $S$ co-evolve in a twodimensional nonlinear dynamical system. Both such lower-dimensional subcases were analyzed in detail in Tramontana et al. (2009). The main findings of such an analysis are summarized in the following section.

\section{Summaries of the 1D and 2D Models}

In this section, we recall the main results regarding the simplified, lower-dimensional subcases analyzed in Tramontana et al. (2009).

\subsection{One-Dimensional Case}

In the absence of interactions, $c^{H}=c^{A}=0$, each market evolves as a one-dimensional dynamical system. Stock markets are represented by simple linear equations and in each of them the unique fundamental steady state is globally stable, at least for reasonable values of the price and demand adjustment parameters. The law of motion of the foreign exchange market is nonlinear, determined by iteration of a cubic map, 
with three fixed points: namely, two non-fundamental steady states, say $P_{1}$ and $P_{2}$, relative to each of the two unimodal branches of the cubic map, surrounding an unstable fundamental steady state $(O)$. The map is symmetric with respect to the fundamental value, which is why the bifurcations involving $P_{1}$ and $P_{2}$ are synchronized. The bifurcation analysis with respect to parameter e highlighted the route to chaotic bull and bear dynamics of the model. The (synchronized) period-doubling bifurcations of $P_{1}$ and $P_{2}$, followed by the usual cascade of flip bifurcations and the homoclinic bifurcations of the two steady states, lead to the coexistence of two symmetric intervals (around $P_{1}$ and $P_{2}$, respectively), each characterized by chaotic dynamics (in the sense of chaos of full measure on an interval). Due to the noninvertibility of the map, within this range of values of parameter $e$ the basins of the two coexisting attractors have a disconnected structure, each being made up of an infinite sequence of intervals which alternate on the real line with that of the competing attractor. For higher values of parameter $e$, the two attractors and their basins merge together via a homoclinic bifurcation of the fundamental steady state $O$. After this point, the exchange rate dynamics, previously confined to below or above the fundamental value, depending on the initial condition, wanders within a unique chaotic interval around the fundamental steady state, alternating bull and bear market episodes in an unpredictable manner. A final bifurcation then occurs when the unique attractor touches the border between its basin and the basin of infinity, $\mathcal{B}_{\infty}$, after which the generic trajectory is divergent.

A crucial tool for the bifurcation analysis, strictly associated with the noninvertibility of the map, is represented by the critical points (local extrema and their iterates), which are at the boundary of chaotic intervals, and their contacts with the unstable steady states.

\subsection{Two-Dimensional Case}

By introducing a partial connection between stock markets $A$ and $H$ (namely, by allowing investors from country $A$ to trade in country $H$ ), the latter turns out to coevolve with the foreign exchange market (whereas market $A$ is still decoupled from the system). As a result, we have a system of two coupled equations, one linear and one nonlinear. In particular, in the nonlinear equation for the exchange rate we also have a feedback from stock market $H$, which makes the dynamics even more intricate. One difference with the one-dimensional case is that now a unique steady state exists for small values of $e$. Another difference is that the symmetry property is lost. Apart from this, in the two-dimensional case we still observe the same multiple steady state structure (when e is large enough) and a similar sequence of local and global bifurcations. More precisely, we highlighted the homoclinic bifurcations that involve the saddle equilibria $P_{1}$ and $P_{2}$ first (albeit now in an asynchronous manner) and then the fundamental equilibrium $O$. Due to this sequence of bifurcations (also called interior and exterior crises in Grebogi et al. (1983), the system has a transition across different dynamic scenarios: from coexisting attracting bull 
and bear chaotic regions, to the disappearance of one of them, to the merging of the two regions into a unique wider chaotic area. The resulting dynamic outcome is a coupled bull and bear market behavior of stock price $H$ and the exchange rate, which may switch across different regions of the two-dimensional phase space with apparently random behavior. In all cases, the bifurcation mechanisms are basically due to contacts ${ }^{3}$ between invariant sets - such as stable manifolds of saddles - and the boundary of chaotic attractors, the latter being made up of portions of critical curves of the noninvertible two-dimensional map (see Mira et al., 1996). Finally, also the bifurcation leading to the disappearance of the unique chaotic attractor is similar to that of the one-dimensional case. Such a two-dimensional analysis has been largely carried out with the help of numerical simulation and graphical visualization. In particular, the tool of the critical curves has suggested how the basins of attraction may acquire a disconnected structure.

\section{Analysis of the 3D Model}

In this section we deal with the complete three-dimensional model, mainly with the help of numerical simulation. Our analysis will show that the dynamic phenomena highlighted in Tramontana et al. (2009) also persist in the full model, and can be detected and understood by extending the approach and techniques used in the lower-dimensional cases to a three-dimensional setup. In particular, we are also able in the full model to detect and explain the sequence of local and global bifurcations that determine the transition between different dynamic regimes: namely, from a unique attracting fundamental equilibrium to coexistence of attracting nonfundamental equilibria, to more complex coexisting attractors, up to the homoclinic bifurcations which bring about a regime of bull and bear market fluctuations, first established by Day and Huang (1990) in a one-dimensional setup, characterized by apparently random switches of prices across different regions of the phase space.

In the full model, stock market traders from countries $A$ and $H$ are allowed to trade in both markets, i.e. $c^{H}>0$ and $c^{A}>0$. In this case, the two stock prices and the exchange rate are all interdependent, and the model has the complete structure (4). The system (4), expressed in deviations ${ }^{4}$ from fundamental values, $x=\left(P^{H}-F^{H}\right), y=\left(S-F^{S}\right)$ and $z=\left(P^{A}-F^{A}\right)$, is represented by a map $T: \mathbb{R}^{3} \rightarrow \mathbb{R}^{3}$ that takes the following form:

\footnotetext{
${ }^{3}$ Following Mira et al. (1996) we call contact bifurcation any contact between two closed invariant sets of different kinds. A contact bifurcation may have several different dynamic effects, depending on the nature of the invariant sets.

${ }^{4}$ Although we work with deviations, in all the following numerical experiments we have checked that original prices never become negative.
} 


$$
T:\left\{\begin{aligned}
x_{t+1}= & x_{t}-a^{H}\left[\left(b^{H}+c^{H}\right) x_{t}+c^{H} \gamma^{H} y_{t}\right], \\
y_{t+1}= & y_{t}-d\left[c^{H}\left(x_{t}+F^{H}\right)\left(x_{t}+\gamma^{H} y_{t}\right)\right. \\
& \left.+c^{A} \frac{z_{t}+F^{A}}{y_{t}+F^{S}}\left(\gamma^{A} \frac{y_{t}}{F^{S}\left(y_{t}+F^{S}\right)}-z_{t}\right)-e y_{t}+f y_{t}^{3}\right], \\
z_{t+1}= & z_{t}-a^{A}\left[\left(b^{A}+c^{A}\right) z_{t}-c^{A} \gamma^{A} \frac{y_{t}}{F^{S}\left(y_{t}+F^{S}\right)}\right] .
\end{aligned}\right.
$$

The model is not tractable analytically. Apart from the fundamental fixed point, say $O=(0,0,0)$, whose existence can be immediately checked, we cannot solve explicitly for the coordinates of further possible non-fundamental equilibria, nor can we obtain easily interpretable analytical conditions for their existence. A brief discussion of the steady states is provided in the following subsection.

\subsection{Fixed Points and Multistability}

By imposing the fixed point condition to (6), we obtain the following system of equations

$$
\begin{gathered}
\left(b^{H}+c^{H}\right) x+c^{H} \gamma^{H} y=0, \\
c^{H}\left(x+F^{H}\right)\left(x+\gamma^{H}\right)+c^{A} \frac{z+F^{A}}{y+F^{S}}\left(\gamma^{A} \frac{y}{F^{S}\left(y+F^{S}\right)}-z\right)-e y+f y^{3}=0, \\
\left(b^{A}+c^{A}\right) z-c^{A} \gamma^{A} \frac{y}{F^{S}\left(y+F^{S}\right)}=0 .
\end{gathered}
$$

We observe from (7) and (9) that any steady state must belong to both the plane of equation:

$$
y=-\frac{x}{q^{H}}
$$

and the surface of equation

$$
z=q^{A} \frac{y}{\left(y+F^{S}\right)}
$$

where

$$
q^{H}:=\frac{c^{H} \gamma^{H}}{b^{H}+c^{H}} ; \quad q^{A}:=\frac{c^{A} \gamma^{A}}{\left(b^{A}+c^{A}\right) F^{S}} .
$$

This implies that when the steady state exchange rate is above the fundamental value $(y>0)$, steady state price $A$ is then above the fundamental value $(z>0)$, whereas steady state price $H$ is below the fundamental value $(x<0)$, and vice versa. From now on, we will label the region $y>0, z>0, x<0$ as the bull region and region $y<0, z<0, x>0$ as the bear region. By substituting (10) and (11) into (8), we are able to express condition (8) in terms of the steady state (deviation of) 
price $H$ only, as follows:

$$
x\left[\frac{f}{\left(q^{H}\right)^{3}} x^{2}+b^{H} x+\left(b^{H} F^{H}-\frac{e}{q^{H}}\right)+M(x)\right]=0,
$$

where

$$
M(x):=b^{A} q^{H} q^{A} \frac{q^{H} F^{S} F^{A}-x\left(q^{A}+F^{A}\right)}{\left(q^{H} F^{S}-x\right)^{3}} .
$$

Therefore, besides the fundamental solution $x=0$, further possible solutions are the roots of the expression in square brackets in (12). Note that for $c^{A}=0$, and therefore $q^{A}=0$ and $M(x)=0$, the $x$-coordinates of further possible steady states are the solutions of a quadratic equation, and their existence was discussed in detail in Tramontana et al. (2009)..$^{5}$

In contrast, if $c^{A}>0$, it becomes impossible to solve (12) analytically. When $c^{A}$ is small enough, we may expect a steady state structure qualitatively similar to that of the two-dimensional subcase $c^{A}=0$, with two further steady states initially appearing simultaneously in the bull region, via saddle-node bifurcation. ${ }^{6}$

However, if $c^{A}$ is large enough, as is the case of the following numerical experiments, as we shall see, it is difficult to detect the appearance of further equilibria and their initial location with respect to the fundamental. We remark that the analytical investigation of the local stability properties of fundamental fixed point $O=(0,0,0)$ is also a difficult task. The Jacobian matrix evaluated at $O$ is given by

$$
J(O):\left[\begin{array}{ccc}
1-a^{H}\left(b^{H}+c^{H}\right) & -a^{H} c^{H} \gamma^{H} & 0 \\
-d c^{H} F^{H} & 1-d\left[c^{H} F^{H} \gamma^{H}+\frac{c^{A} F^{A} \gamma^{A}}{\left(F^{S}\right)^{3}}-e\right] & \frac{d c^{A} F^{A}}{F^{S}} \\
0 & \frac{a^{A} c^{A} \gamma^{A}}{\left(F^{S}\right)^{2}} & 1-a^{A}\left(b^{A}+c^{A}\right)
\end{array}\right]
$$

and its eigenvalues (which are roots of a third-order polynomial) cannot be solved for explicitly. Nor can we write down tractable analytical conditions for the eigenvalues to be smaller than one in modulus. We shall now study the local and global bifurcations via numerical investigation, supported by our knowledge of the model behavior in the simplified, two-dimensional case. In fact, as we shall see, the analysis performed in Tramontana et al. (2009) provides important guidelines for understanding the dynamic phenomena occurring in this more complex three-dimensional model.

With the parameter setting used in following numerical simulations (as well as with other similar constellations of parameters) we do not observe the appearance of the non-fundamental equilibria via saddle-node bifurcation. Instead, a pitchfork

\footnotetext{
${ }^{5}$ Moreover, in this case, in which market A decouples from the system, (11) reduces to $z=0$.

${ }^{6}$ This is also confirmed by numerical simulations.
} 
bifurcation of the fundamental steady state seems to occur, leading to the appearance of two new stable equilibria, one in the bull region and one in the bear region, at the same parameter value at which the fundamental becomes unstable. ${ }^{7}$ The situation resulting from the local bifurcation of the fundamental steady state is in any case qualitatively the same as for the two-dimensional subcase. That is, the phase space is shared amongst the basins of attraction of two non-fundamental equilibria, separated by the stable set of the (saddle) fundamental steady state. From now on, the bifurcation sequences involving the two coexisting equilibria (or, more generally, the two coexisting attractors) follow paths similar to those observed in the two-dimensional model, albeit involving stable and unstable manifolds in higher dimensions. In this paper we confirm and strengthen almost all of the results of the two-dimensional case, albeit via numerical simulations only. We shall describe various kinds of homoclinic bifurcations, following the same scheme of the study carried out in Tramontana et al. (2009).

Our base parameter selection is the following: $a^{H}=0.41, b^{H}=0.11, c^{H}=0.83$, $F^{H}=4.279, \gamma^{H}=0.3, d=0.35, f=0.7, F^{S}=6.07$ (which are the same parameters used in the simulations in Tramontana et al. (2009), enabling a direct comparison), $a^{A}=0.43, b^{A}=0.21, c^{A}=0.9, \gamma^{A}=0.36$ and $F^{A}=1.1$. In order to sufficiently distinguish the model from the two-dimensional case studied in Tramontana et al. (2009) (where $c^{A}$ is zero), we have chosen a value of $c^{A}$ that is much further away from zero, and even higher than $c^{H}$. Bifurcations similar to those described below are observed with several other parameter constellations. The numerical analysis performed in the Appendix shows that $O$ loses stability when one eigenvalue becomes equal to 1 at $e \simeq 0.125$. We argue that this corresponds to a pitchfork bifurcation, because we observe the simultaneous appearance of two further equilibria, which we denote as $P_{1}$ (in the bear region) and $P_{2}$ (in the bull region). Figure 1 shows the asymptotic dynamics in the three-dimensional phase space for increasing values of $e$. We can see that the fundamental fixed point is unstable and that two new stable fixed points exist. The stable fixed points are located on opposite sides with respect to plane $y=0$ (i.e. $S=F^{S}$ ), as shown in Fig. 1a. Since only one eigenvalue of $J(O)$ becomes larger than 1, while two other eigenvalues are real and still smaller than one in absolute value, the fundamental equilibrium is a saddle with a one-dimensional unstable manifold (made up of two branches, connecting $O$ with $P_{1}$ on one side and $O$ with $P_{2}$ on the opposite side), while the stable set $W_{O}^{S}$ of the origin is a two-dimensional manifold, which separates the two basins of attraction of the two coexisting fixed points. In other words, the frontier of the basins of attraction of $P_{1}$ and $P_{2}$, say $\mathcal{B}_{1}$ and $\mathcal{B}_{2}$, respectively, includes surface $W_{O}^{s}$.

Moreover, it is easy to see that divergent behavior is also possible, so that the basin of divergent trajectories, $\mathcal{B}_{\infty}$, also exists (and will be involved in the final bifurcation, as we shall see below). The structure of the basins after the appearance

\footnotetext{
${ }^{7}$ We remark that this is just numerical evidence, and we cannot exclude the existence of a sequence similar to that described for the 2D model (i.e. a Saddle-Node bifurcation immediately followed by a Transcritical), occurring in a very narrow range of parameter $e$.
} 


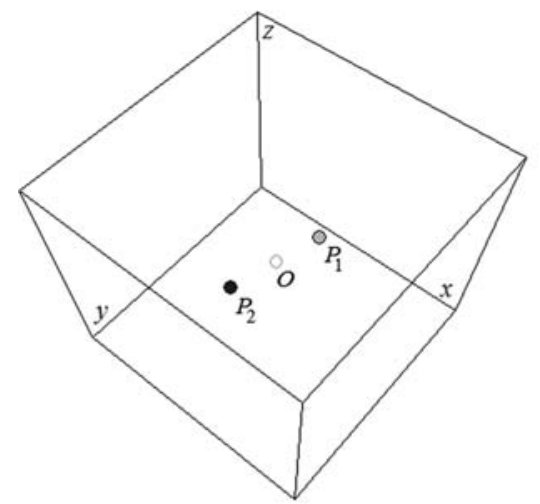

(a)

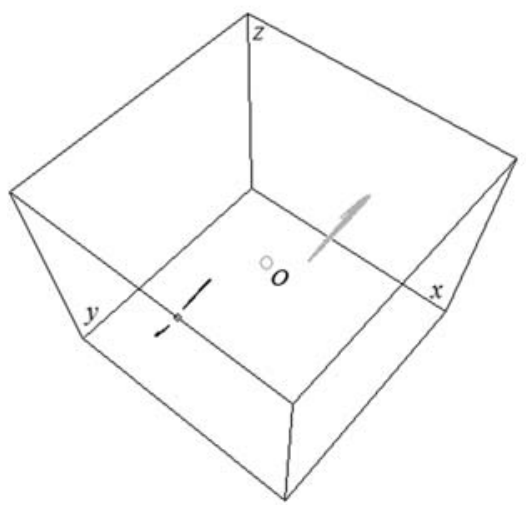

(c)

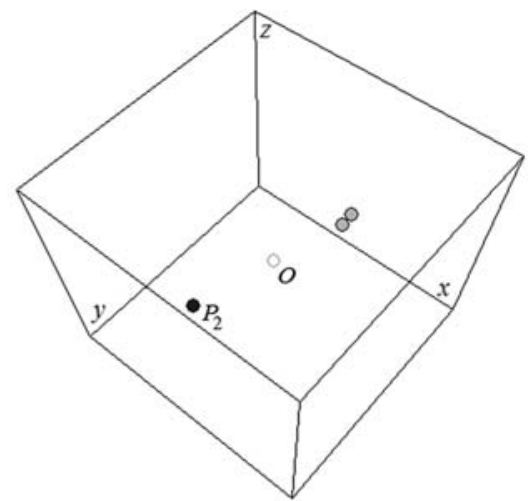

(b)

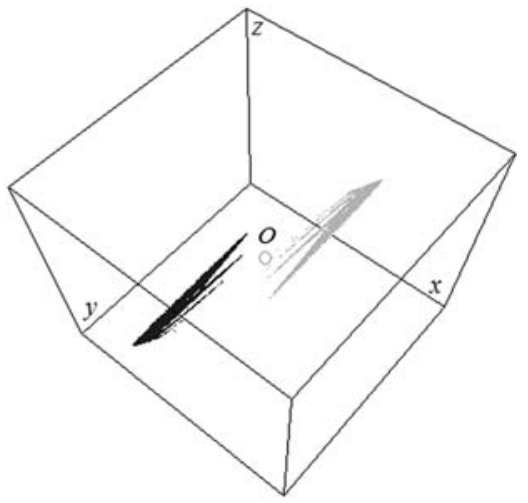

(d)

Fig. 1 Coexisting attractors for increasing values of parameter e and other parameters according to our base selection. In (a), $e=0.89$, the attractors are two stable fixed points $P_{1}$ and $P_{2}$. In (b), $e=$ 2.43, there is coexistence of the stable fixed point $P_{2}$ and a stable 2-cycle. In $(\mathbf{c}), e=3.576$, one chaotic attractor (blue, in the bear region) consists of a unique piece (after the homoclinic bifurcation of the fixed point $P_{1}$ ) while the other chaotic attractor (red, in the bull region) is made up of two disjoint pieces, on opposite sides with respect to the fixed point $P_{2}$. In $(\mathbf{d}), e=4.1841$, both have become one-piece attractors, and the light grey one approaches the stable set of the fundamental fixed point in the origin. The boxes are centered in $O$ and the range of all axes is $[-3,+3]$

of the two new attractors is shown in Fig. 2, where a cross section of a plane through the fundamental fixed point $O=(0,0,0)$ is considered. The value of the parameter is $e=0.89$, as in Fig. 1a, and two attracting fixed points coexist. In the cross section, the different grey tonalities belong to different basins of attractions. We denote in grey the basin of the fixed point $P_{1}$ (in the bear region). The basin of fixed point $P_{2}$ (in the bull region) is light grey, while points generating divergent trajectories, and thus belonging to the basin $\mathcal{B}_{\infty}$, are shown in dark grey. 


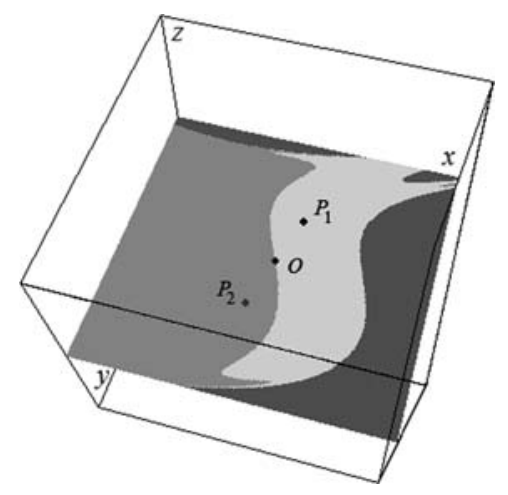

Fig. 2 Cross section along a plane through the fundamental fixed point $O=(0,0,0)$. The value of parameter is $e=0.89$, as in Fig. 1a. The box is centered in $O$ and the range of axes is $[-3,+3]$ for all state variables. In the cross section, different colors denote different basins of attractions. Basin $\mathcal{B}_{1}$ of the fixed point $P_{1}$ is in grey, basin $\mathcal{B}_{2}$ of the fixed point $P_{2}$ is in light grey, basin $\mathcal{B}_{\infty}$ is in dark gray

As already conducted in Tramontana et al. (2009) of our study, we analyze the sequence of bifurcations occurring when parameter $e$ is increased. We first show a bifurcation diagram of the asymptotic behavior of state variable $y$ as a function of parameter $e$. The diagram (Fig. 3) highlights how a sequence of bifurcations very similar to those observed in the two-dimensional case also occurs in the threedimensional case and, as expected, in an asynchronous manner (because in the full model, as well as in the two-dimensional subcase studied in Tramontana et al., 2009, there is no symmetry with respect to the origin). In Fig. 3a, the initial condition is taken close to the fixed point $P_{1}$, while in Fig. $3 \mathrm{~b}$ the starting point is close to the other fixed point $P_{2}$. The global bifurcations first involve the attractor associated with equilibrium $P_{1}$ (in blue) and then that associated with $P_{2}$ (in red).

\subsection{Homoclinic Bifurcation of Equilibria $P_{1}$ and $P_{2}$}

As noted above, after their appearance, both locally stable fixed points undergo a cascade of flip bifurcations (in an asynchronous manner), leading to chaos (see Figs. 3 and 1). In particular, in Fig. 1c we can see that the attracting set in the bull region (in red) is still made up of two disjoint pieces, located on opposite sides with respect to the unstable fixed point $P_{2}$, while the second attractor, located in the bear region (in blue), is already a one-piece chaotic attractor. Although we do not have the coordinates of the unstable fixed points $P_{1}$ and $P_{2}$, we can state that in this case (Fig. 1c) fixed point $P_{1}$ is already homoclinic, at least on one side, because it belongs to the invariant chaotic area (and it is probably located on its boundary, as it occurs in the 2D model), while the fixed point $P_{2}$ is not yet homoclinic (because it is not jet included in the chaotic area), although it will be involved later 

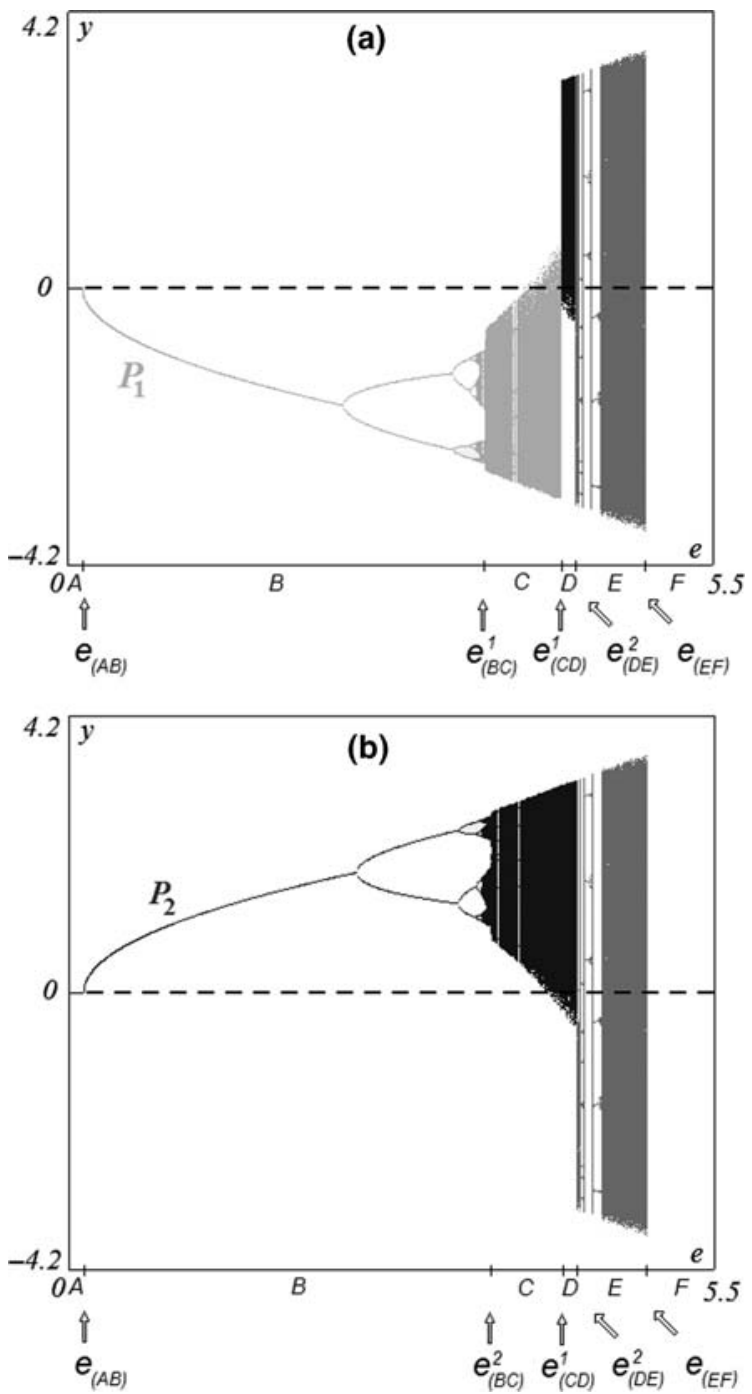

Fig. 3 Bifurcation diagrams of $y$ vs. parameter $e$, ranging from 0 to 5.5. The range of $e$ is subdivided into different intervals. In interval $A$ the only attractor is the fundamental equilibrium $O$. Its pitchfork bifurcation occurs at $e \simeq 0.125$, after which two new stable equilibria appear. In (a) the initial condition is close to the fixed point $P_{1}$, in (b) it is close to $P_{2}$. In interval $B$ we observe a complete route to chaos for each fixed point. The homoclinic bifurcation of $P_{1}$ occurs at $e_{(B C)}^{1} \simeq 3.56$, which results in the one-piece chaotic attractor in light grey. The homoclinic bifurcation of $P_{2}$ occurs at $e_{(B C)}^{1} \simeq 3.6$, leading to the one-piece chaotic attractor in dark grey. In interval $C$ there is coexistence of two one-piece chaotic attractors. The upper bound of interval $C$ corresponds to a homoclinic bifurcation of the origin. In (a) the light grey chaotic attractor disappears at the first homoclinic bifurcation of the origin, which occurs at $e_{(C D)}^{1} \simeq 4.185$, so that for any $e$ in interval $D\left(e_{(C D)}^{1}<e<e_{(D E)}^{2}\right)$ the unique attractor is the dark grey one. The second homoclinic bifurcation of the origin occurs at $e_{(D E)}^{2} \simeq 4.3$ and leads to an explosion of the chaotic attractor into a wider region (in grey). This unique chaotic attractor exists up to its final bifurcation at $e_{f} \simeq 5.03$ 
(i.e. for larger $e$ ) in a homoclinic bifurcation, causing the reunion of the two pieces of chaotic attractor around $P_{2}$. Figure $1 \mathrm{~d}$ indeed shows the situation existing after both the first homoclinic bifurcations of equilibria $P_{1}$ and $P_{2}$ have occurred.

In the bifurcation diagram in Fig. 3 we plot the asymptotic behavior of the state variable $y$, as e varies in the range $[0,5.5]$. In the interval of values of $e$ denoted by $A$, the only attracting set is the fundamental equilibrium $O$. Its pitchfork bifurcation occurs at $e \simeq 0.125$, after which we have the appearance of two further stable equilibria. In (a) the initial condition is taken close to the fixed point $P_{1}$, while in (b) it is taken close to the fixed point $P_{2}$. The fixed points are stable up to their flip bifurcation, which occurs for $P_{1}$ first, and then for $P_{2}$. In the interval denoted by $B$ we observe the typical route to chaos for each fixed point, and the parameter values $e_{(B C)}^{1}$ and $e_{(B C)}^{2}$ are the homoclinic bifurcation values of $P_{1}$ and $P_{2}$, respectively, at which the reunion of two pieces into one single chaotic attractor takes place. In the proposed example, we first observe this bifurcation in the bear region, at $e_{(B C)}^{1} \simeq 3.56$ (leading to the one-piece chaotic attractor in blue), and then in the bull region, at $e_{(B C)}^{2} \simeq 3.6$ (leading to the one-piece chaotic attractor in red).

The coexistence of two disjoint attractors in the bull and bear regions is coupled with an increase of complexity in the structure of the related basins of attraction $\mathcal{B}_{1}$ and $\mathcal{B}_{2}$. An example is shown in Fig. 4: in (a) we show the two disjoint attractors and in (b) a cross section through the origin shows the basins in different colors. $\mathcal{B}_{1}$, in pink, is the locus of initial points converging to the chaotic attractor in blue, and $\mathcal{B}_{2}$, in orange, is the locus of points converging to the chaotic attractor in red, while the gray points belong to basin $\mathcal{B}_{\infty}$. Note that the basins are now disconnected: within the region that approximately coincides with basin $\mathcal{B}_{1}$ of Fig. 2 there are now also points belonging to basin $\mathcal{B}_{2}$ and to $\mathcal{B}_{\infty}$; at the same time, points belonging to basin $\mathcal{B}_{1}$ and to $\mathcal{B}_{\infty}$ are now located in the region previously belonging to basin $\mathcal{B}_{2}$ in Fig. 2. This phenomenon is again due to contact bifurcations of the basins of attraction with critical sets (critical surfaces, in this three-dimensional case). That is, denoting by $J(x, y, z)$ the Jacobian matrix of the map (6) and by $S C_{-1}$ the locus
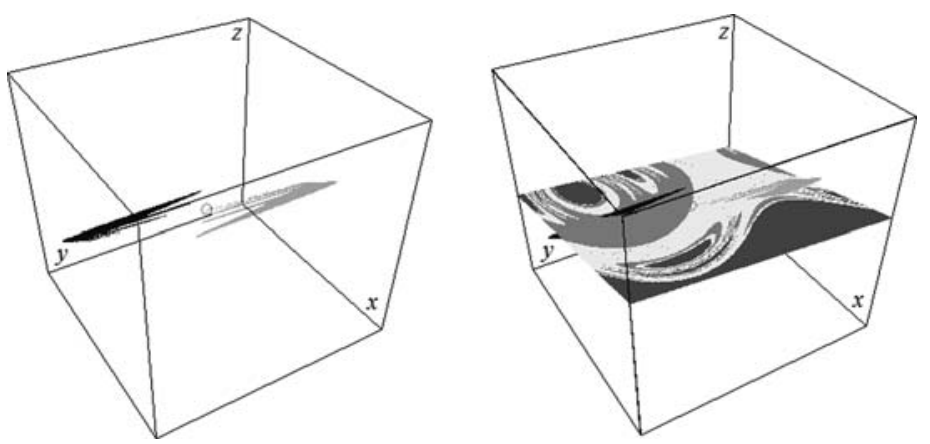

Fig. 4 Coexisting attractors at $e=4.1841$. The boxes are centered in $\mathrm{O}$ and the range of axes is $[-3,+3]$. In (b) a plane through the origin $O$ is shown, along which different colors denote different basins of attraction, as in Fig. 2 
of points defined by the equation det $J(x, y, z)=0$, this set plays the same role of the critical points $x_{-1}^{m}$ and $x_{-1}^{M}$ in the one-dimensional map corresponding to the subcase $c^{H}=c^{A}=0$, and to the critical curve $L C_{-1}$ in the two-dimensional map corresponding to $c^{A}=0$, both analyzed in Tramontana et al. (2009). The image of $S C_{-1}$ under map $T$ gives a surface, $S C:=T\left(S C_{-1}\right)$, which is responsible for the contact bifurcations of the basins of attraction. In the $3 \mathrm{D}$ phase space this critical surface $S C$ separates regions of points with a different number of rank-1 preimages. When basin $\mathcal{B}_{1}$ (or basin $\mathcal{B}_{\infty}$ ) touches the critical surface $S C$ and then crosses it, a portion of the basin, say $H^{\prime}$, enters a region of the phase space whose points have a higher number of preimages, thus leading to the appearance of new portions of the basin. Such portions consist of the region (volume) $T^{-1}\left(H^{\prime}\right)$, located around the critical surface $S C_{-1}$, and of its further preimages.

\subsection{Homoclinic Bifurcation of the Fundamental Equilibrium $O$}

The coexistence of two attractors located in two disjoint bull and bear regions ends at the first homoclinic bifurcation of the origin. The section of the basins of attractions in Fig. 4b shows that the chaotic attractor in the bear region, colored blue, is very close to the boundary of its basin $\mathcal{B}_{1}$. Moreover, we know that the frontier of the two basins $\mathcal{B}_{1}$ and $\mathcal{B}_{2}$ includes the two-dimensional stable set $W_{O}^{S}$ of the fundamental fixed point $O$, which is now a set with a complex structure. Thus, from the closeness of the chaotic area to the origin we can argue that in the parameter situation shown in Fig. 4b we are already very close to this first homoclinic bifurcation of the origin (while the second one is due to the other chaotic attractor, which is still far from the origin).

At the fixed point itself the stable set $W_{O}^{S}$ is a surface tangent to the plane generated by the eigenvectors associated with the two stable eigenvalues of the Jacobian matrix $J(O)$. A portion of this plane (tangent at the origin to the surface $W_{O}^{s}$ ) is shown in Fig. 5, at $e=4.1841$ (same parameter value as in Fig. 4). At this value the

Fig. 5 Coexisting attractors for $e=4.1841$ (as in Fig. 4) and a portion of the plane through the origin $O$, tangent to the stable set $W_{O}^{S}$

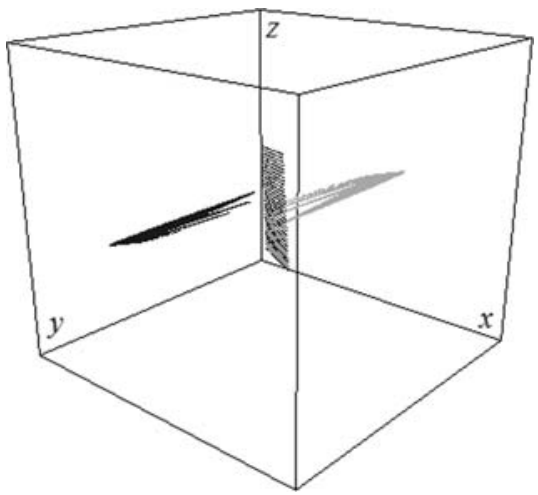


eigenvalues of the Jacobian matrix $J(O)$ are given by $\xi_{1}=2.1725, \xi_{2}=-0.5341$ and $\xi_{3}=0.5216$. The eigenvectors associated with eigenvalues $\xi_{2}$ and $\xi_{3}$ (less than 1 in modulus), say $v_{2}$ and $v_{3}$, respectively, are given by

$$
v_{2}=\left(\begin{array}{c}
-0.6066 \\
-0.769 \\
-0.2017
\end{array}\right), \quad v_{3}=\left(\begin{array}{c}
-0.2582 \\
-0.2836 \\
-0.9235
\end{array}\right)
$$

and the tangent plane is generated by these two vectors. We can see that in Fig. 5 the tangent plane is already crossed by the chaotic attractor in blue. This means that we are not far from the parameter value at which a contact with surface $W_{O}^{S}$ occurs. Since one branch of the unstable set $W_{O}^{u}$ of the origin tends to the chaotic attractor, the contact of the chaotic attractor with the stable set of the origin is also a contact between the unstable set $W_{O}^{u}$ and the stable set $W_{O}^{S}$, leading to the first homoclinic bifurcation of the fixed point $O$.

After the contact, the stable and unstable sets have infinitely many transverse intersections. However, the chaotic set associated with the origin is not observable in the asymptotic dynamics. In fact, as a result of this bifurcation we have the disappearance of the chaotic attractor in the bear region. That is, the previous light grey chaotic attractor has now turned into a chaotic repeller, which also includes homoclinic trajectories on one side of the origin. We recall that a contact bifurcation causing the disappearance of a chaotic attractor always lives a chaotic repeller in its place in the phase space. This chaotic repeller is formed by all the unstable cycles previously existing in the chaotic set, and the related stable sets, or insets (see Mira et al., 1996 for further details). Thus although in Fig. 6a we observe only one attractor on one side of the stable set of the origin, we know that on the

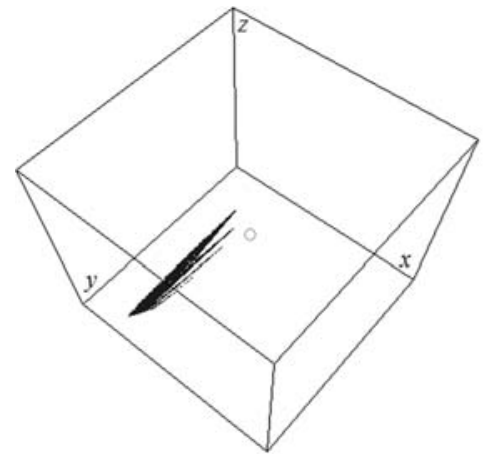

(a)

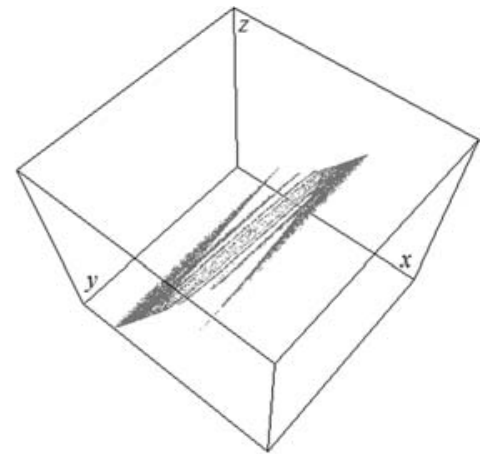

(b)

Fig. 6 (a) Unique chaotic set in the bull region, at $e=4.208$, after the first homoclinic bifurcation of the fundamental equilibrium $O$. (b) Unique chaotic set covering both the bull and bear regions, at $e=4.761$, after the second homoclinic bifurcation of the fundamental equilibrium $O$. Boxes are centered in $O$ and the range of axes is $[-3,+3]$ 
other side a chaotic repeller exists, and its existence may be put in evidence when a contact between the existing chaotic attractor and the origin occurs (at another homoclinic bifurcation of the origin). We may observe the chaotic repeller via the long chaotic transient of trajectories starting from initial conditions in the old $\mathcal{B}_{1}$ area: they remain in the old region for several iterations before converging to the chaotic set in the bull area.

For this reason, the interval labelled $C$ in Fig. 3a (where two one-piece chaotic attractors coexist) ends with the first homoclinic bifurcation of the origin, which occurs at $e_{(C D)}^{1} \simeq 4.185$, when the chaotic attractor in the bear region disappears and the generic initial condition in that region then converges to the dark grey attractor, in the bull region. Similarly to the two-dimensional case, a range of values of the parameter $e$ exists such that the chaotic attractor located (approximately) in the region $S>F^{S}(y>0)$, colored dark grey, becomes the only attractor in the phase space (see Fig. 6a). From the asymptotic behavior, shown in Fig. 6a, we cannot observe the chaotic repeller, which we know exists. The chaotic repeller will again be observable after the second homoclinic bifurcation of the origin, which occurs at $e_{(D E)}^{2} \simeq 4.3$, leading to an explosion of the chaotic attractor into a wide region of the phase space, as shown in Fig. 6 b.

From Fig. 6a we can see that the tongues of the dark grey chaotic set increasingly approach the fundamental fixed point, and thus we are very close to the second homoclinic bifurcation of $O$. This bifurcation involves a contact between the branch of $W_{O}^{u}$ that converges to the chaotic attractor in the bull region and the surface representing the stable set $W_{O}^{s}$. The result of this bifurcation is an explosion of the chaotic set (which includes both the previous chaotic attractor and the previous chaotic repeller), as shown in Fig. 6 b.

This brings about a major change of the dynamics. Whatever the initial condition is (from either the bear or the bull region), the trajectory will wander in both regions, jumping from one to the other after an unpredictable number of iterations. An example of the resulting fluctuations of the state variables is given in Fig. 7. The dynamics we obtain are much more intricate than those observed in Day and Huang (1990). The reason for this is that there is a feedback process from the foreign exchange markets on the stock markets and from the stock markets on the foreign exchange market. The first feedback process generates endogenous dynamics in the stock markets, where otherwise no dynamics would be observable.

The second feedback process may be interpreted as deterministic noise impacting on the evolution of the exchange rate. Also, the three markets demonstrate excess volatility and endogenous bubbles and crashes.

\subsection{Final Bifurcation}

After the above-described second homoclinic bifurcation of the origin, the region of the phase space covered by the chaotic dynamics becomes wider as parameter e increases. The oscillations of the trajectories increase in amplitude, and we approach 

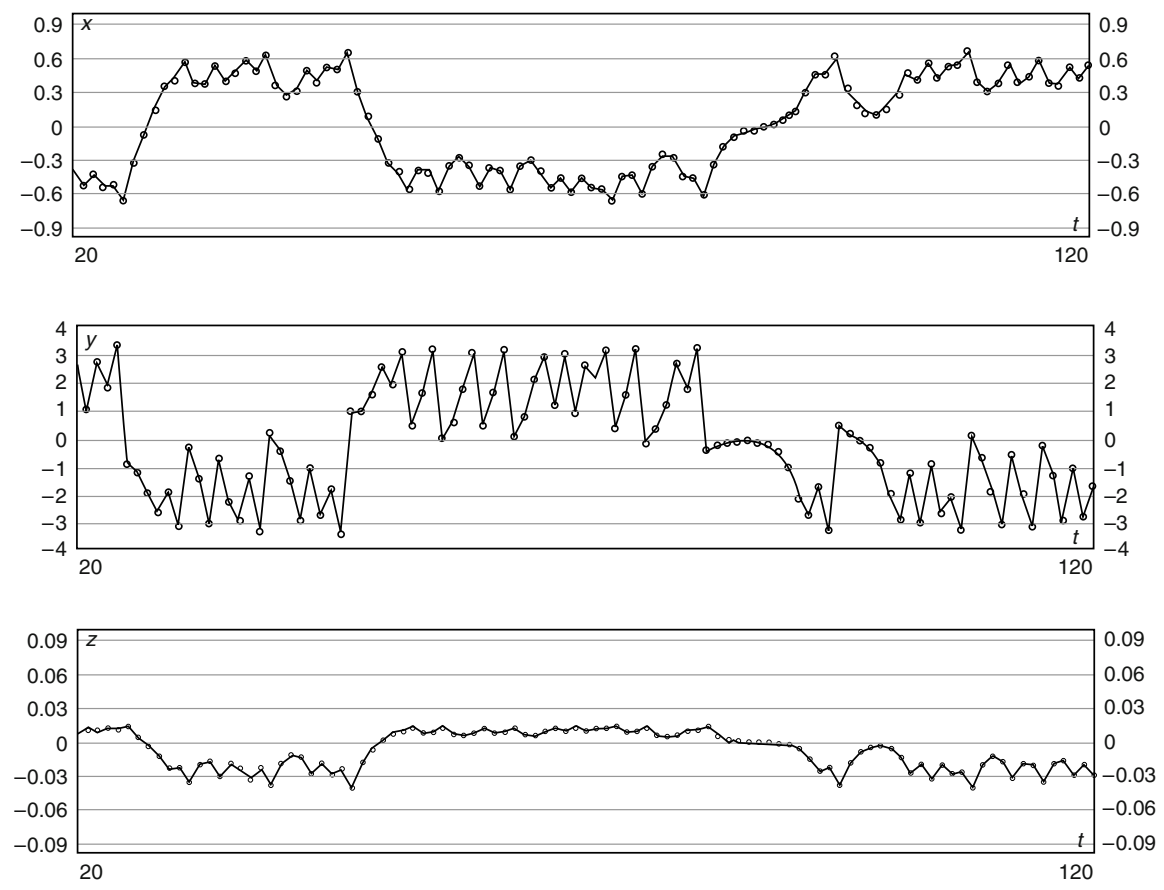

Fig. 7 Trajectories of the state variable $x$ (deviation of stock price $P^{H}$ from the fundamental price $F^{H}$ ), $y$ (deviation of the exchange rate $S$ from the fundamental exchange rate $F^{S}$ ), $z$ (deviation of stock price $P^{A}$ from the fundamental price $F^{A}$ ), obtained at $e=4.75$

a catastrophic situation, after which trajectories will be mainly divergent. In Fig. 3 this bifurcation is revealed by the existence of a unique attractor, colored grey, which covers both chaotic regions and exists up to its final bifurcation at $e_{f} \simeq 5.03$. The final bifurcation is again given (as in the one- and two-dimensional cases studied in Tramontana et al., 2009) by a contact of the chaotic attractor with the frontier of its basin of attraction. We recall (see Mira et al., 1996) that a contact between an invariant set and the basin of divergent trajectories always leads to a final bifurcation, because the invariant set will no longer exist after the contact, and almost all the points whose trajectory was previously trapped into the invariant set will then have divergent trajectories. In our example this is shown in Fig. 8 where, for a specific value of e close to the final bifurcation, we represent the attractor in black and its basin of attraction in light grey. ${ }^{8}$ Dark gray points, as usual, denote points belonging

\footnotetext{
${ }^{8}$ For a better visualization, the region of the three-dimensional phase space represented in Fig. 8 also includes a set of points that are not economically meaningful (the bottom part of the cube and of the related sections), but the attractor and the contacts that give rise to the final bifurcation all belong to the economically relevant zone.
} 

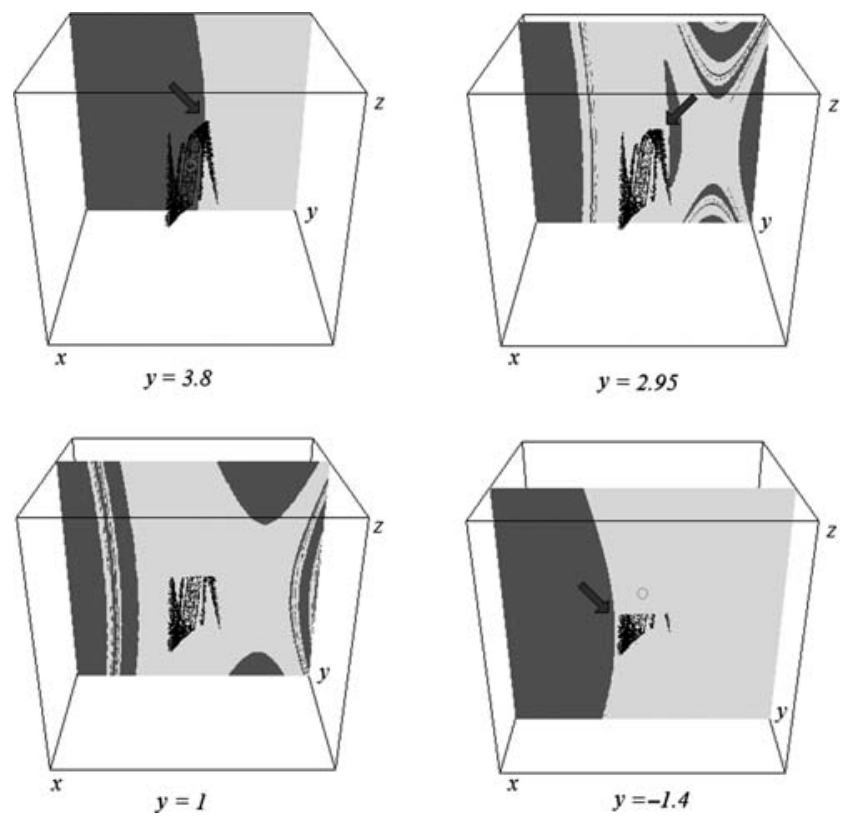

Fig. 8 Chaotic attractor at $e=5.02$ and four different sections of the three-dimensional phase space through planes of equation $y=k$. The dark gray points belong to $\mathcal{B}_{\infty}$ and thus generate divergent trajectories; the light grey points belong to the basin of attraction of the attracting set (in black). The bottom part of each section corresponds to initial conditions that have no economic relevance $\left(z<-F^{A}\right)$, included only for better visualization of the basins. The contacts occur in the meaningful region

to basin $\mathcal{B}_{\infty}$. Figure 8 shows four different sections with planes of equation $y=k$. In the first hyperplane (at $y=3.8$ ), the boundary between the light and dark gray points is a simple line, and this section is still far from the chaotic attractor. In the second cross section (at $y=2.95$ ), the boundary has become more complex, and the attractor is crossed: the section of attractor belonging to the plane, still inside the light grey area, is close to the frontier, in the points indicated by an arrow. In the third section (at $y=1$ ), the attractor again appears a long distance from the border of the basin. Finally, the last section (at $y=-1.4$ ) again suggests that a portion of the attractor is close to the frontier, in the points indicated by the arrow.

The contact between two invariant sets of different nature (the chaotic attractor and the frontier of its basin) leads to the final bifurcation, which will leave a chaotic repeller instead of the chaotic attractor. That is, after this final bifurcation the model is no longer meaningful, as the generic trajectory in the phase space is a divergent trajectory (maybe after a long chaotic transient). The chaotic repeller survives in a set of zero measure, almost inaccessible, and includes all of the unstable fixed points and cycles, as well as all of their stable sets. 


\section{Conclusions}

In this paper we have furthered the study of a dynamic model started in Tramontana et al. (2009), where two stock markets are linked with each other due to the trading activity of foreign investors. Connections occur through the foreign exchange market, where demand for currencies, and consequent exchange rate adjustments, are generated partly by international stock market transactions and partly by the trading activity of heterogeneous foreign exchange speculators. The model results in a three-dimensional nonlinear dynamical system, which is able to generate the typical bull and bear dynamic behavior already detected and discussed by Day and Huang (1990) in a one-dimensional financial market model with fundamentalists and chartists. The previous study was mainly devoted to the derivation of the model and a thorough analysis of its dynamic behavior in simplified one- and two-dimensional cases, corresponding to situations in which the three markets are at least partially disconnected, due to restrictions to the trading activity of foreign investors.

This present paper is focused on the dynamic behavior of the complete threedimensional model. We have presented a study of the full model carried out mainly by numerical simulation and graphical visualization, suitable to reveal contact bifurcations between invariant sets of different nature. Following the road map provided by the analysis performed in Tramontana et al. (2009), and taking advantage of the techniques employed in the 1D and 2D cases, we have seen that the homoclinic bifurcations also occur in this complete model. Thus, as expected, also in the 3D case it turns out that the typical bull and bear dynamics - with seemingly random switches of stock prices and exchange rates across different regions of the phase space - result from a sequence of global bifurcations involving both the nonfundamental steady states and the fundamental equilibrium of the model. Our results thus extend such dynamic mechanisms, which provide a simplified yet intriguing explanation for the emergence of bubbles and crashes in financial markets, to higher-dimensional setups.

\section{Appendix}

Given the parameters selection used in this work (i.e. $a^{H}=0.41, b^{H}=0.11$, $c^{H}=0.83, F^{H}=4.279, \gamma^{H}=0.3, d=0.35, f=0.7, F^{S}=6.07, a^{A}=0.43$, $\left.b^{A}=0.21, c^{A}=0.9, \gamma^{A}=0.36, F^{A}=1.1\right)$, from (13) the Jacobian matrix of the three-dimensional map evaluated at the fixed point $O=(0,0,0)$ becomes

$$
J(O):\left[\begin{array}{ccc}
0.6146 & -0.10209 & 0 \\
1.2430495 & 0.6265274+0.35 e & 0.057084 \\
0 & 0.003781256 & 0.05227
\end{array}\right]
$$


so that we look for the necessary and sufficient conditions for $O$ to have all the eigenvalues less than one in modulus, roots of the characteristic polynomial

$$
\lambda^{3}+A_{1} \lambda^{2}+A_{2} \lambda+A_{3}=0,
$$

where

$$
\begin{aligned}
& A_{1}=-1.7638-0.35 e, \\
& A_{2}=0.9067+0.398055 e, \\
& A_{3}=-0.1348-0.1124 e .
\end{aligned}
$$

Following Farebrother (1973) the eigenvalues of the polynomial given above have to satisfy the following conditions (equivalent conditions can be found in Gandolfo, 1980, Yury's conditions in Elaydi, 1970 and Okuguchi and Irie, 1990):

$$
\begin{aligned}
& \text { (i) } 1+A_{1}+A_{2}+A_{3}>0, \\
& \text { (ii) } 1-A_{1}+A_{2}-A_{3}>0, \\
& \text { (ii i) } 1-A_{2}+A_{1} A_{3}-\left(A_{3}\right)^{2}>0, \\
& \text { (iv) } A_{2}<3 .
\end{aligned}
$$

In our case condition $(i)$ is satisfied for $e<0.125$ (approximate value). Condition ( $i$ i ) becomes $3.8053+0.8605 e>0$ and is obviously satisfied for positive values of $e$. Condition ( $i$ i $i$ ) is satisfied for $e<3.3096$ and $e>3.54$, while condition ( $i v$ ) is satisfied for $e<5.258821$. Starting from values of the parameter e positive and close to 0 and increasing its value, the first condition which is violated is $(i)$, so that $e=0.125$ is the bifurcation value at which the fixed point loses stability.

\section{References}

Day, R. H., \& Huang, W. (1990). Bulls, bears and market sheep. Journal of Economic Behavior and Organization, 14, 299-329.

Elaydi, S. N. (1970). An introduction to difference equations. New York: Springer.

Farebrother, R. W. (1973). Simplified Samuelson conditions for cubit and quartic equations. The Manchester School of Economic and Social Studies, 41, 396-400.

Gandolfo, G. (1980). Economic dynamics: Methods and models. Amsterdam: North Holland.

Grebogi, C., Ott, E., \& Yorke, J. A. (1983). Crises, sudden changes in chaotic attractors, and transient chaos. Physica D, 7, 231.

Mira, C., Gardini, L., Barugola, A., \& Chatala, J. C. (1996). Chaotic dynamics in two-dimensional noninvertible maps. Singapore: World Scientific.

Okuguchi, K., \& Irie, K. (1990). The Shur and Samuelson conditions for a cubic equation. The Manchester School of Economic and Social Studies, 58, 414-418.

Tramontana, F., Gardini, L., Dieci, R., \& Westerhoff, F. (2009). The emergence of "bull and bear" dynamics in a nonlinear model of interacting markets. Discrete Dynamics in Nature and Society, 2009, 310471. 